\title{
Schilddrüsenoperation wegen Hakenkreuz-Tattoo abgebrochen
}

\section{In Paderborn hat sich ein jüdischer Arzt geweigert, einem Mann mit Hakenkreuz-Tattoo die Schilddrüse zu entfernen. Das durfte er auch, sagt die Ärztekammer.}

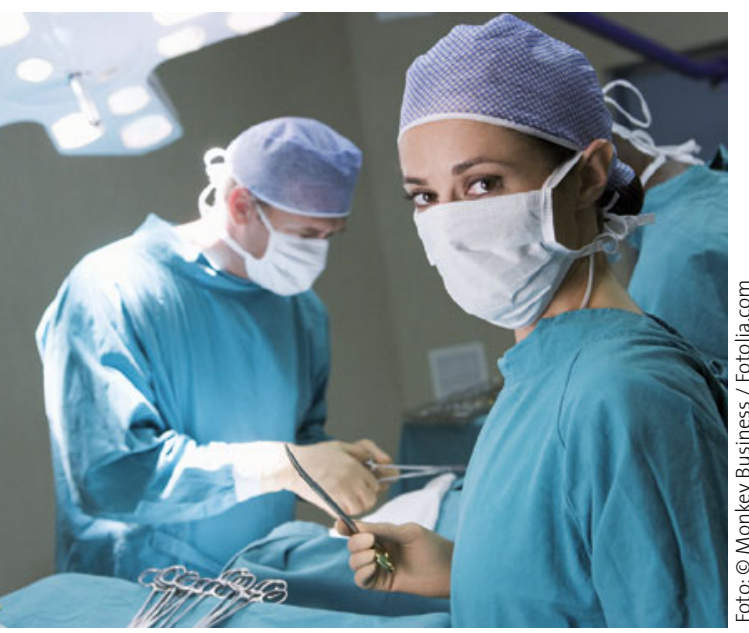

Was soll oder darf der Arzt, wenn ihm sein Patient nicht "geheuer" ist?
- Im katholischen Brüderkrankenhaus St. Josef in Paderborn hat sich ein jüdischer Arzt geweigert, einen Mann zu operieren, der ein großes Hakenkreuz auf dem Oberarm eintätowiert hatte. Der 36-Jährige lag bereits in Narkose, ihm sollte die Schilddrüse entfernt werden.

Der Mediziner informierte die Ehefrau, dass es ihm unmöglich sei, ihren Mann zu operieren. Die Chefärztin des Krankenhauses übernahm den Eingriff. Die empörte Ehefrau setzte offenbar die "Bild-Zeitung" von dem Fall in Kenntnis, das "Westfalen-Blatt" nahm die Sache dann auf.

\section{Ärztekammer:} ein ,außergewöhnlicher Vorgang“" „Bei uns ist bislang keine Beschwerde eingegangen", sagt der Sprecher des Ärztekammer Westfalen-Lippe (ÄKWL) Volker Heiliger. Er bezeichnete den Vorgang als „außergewöhnlich“. Die
Kammer müsse in solchen Fällen prüfen, ob sich der Arzt einer Berufsrechtsverletzung schuldig gemacht habe. Eine solche sieht die Rechtsabteilung der ÄKWL in diesem Fall nicht. Zwar müssten Ärzte in der Regel ohne Ansehen der Person helfen. Es könnten aber „schwer wiegende Rechtfertigungsgründe" vorliegen, die dem Arzt ein Abweichen von diesem Grundsatz ermöglichen, sagt Heiliger. „Die liegen in diesem Fall offenbar vor."

Das Krankenhaus habe zudem umgehend für die Versorgung des Patienten gesorgt. „Es handelte sich nicht um einen Notfall, der Mann war nie in Gefahr", sagt Heiliger. Das bestätigt eine Sprecherin des Krankenhauses. Sie betont, dass sich der Arzt korrekt verhalten habe. Weitere Auskünfte zu dem Vorgang gibt sie nicht. Die Klinik will die Privatsphäre des Arztes schützen.

ILSE SCHLINGENSIEPEN .

\section{AKR: Die Kodierung dauert zu lange}

\section{Die Einführung der Ambulanten Kodierrichtlinien (AKR) löst weiter heftige Diskussionen aus. Neben der KV Hessen kämpfen nun auch die Landesverbände Hessen und Rhein- land-Pfalz des Hausärzteverbandes gegen die Einführung.}

- In einem Rundschreiben des Landesverbands Rheinland-Pfalz heißt es, dass die Umsetzung der AKR für die Ärzte je nach Umfang der Kodierung einen Zeitaufwand von zwei bis fünf Minuten pro Patient bedeutet. Deshalb will sich der Verband dafür einsetzen, dass die AKR in Rheinland-Pfalz nicht eingeführt werden oder dass zumindest ausreichend lange Übergangsfristen eingerichtet werden.

Auch hat der Hausärzteverband in Rheinland-Pfalz eine Arbeitsgruppe eingerichtet, „die geeignete Instrumente entwickeln wird, um in unseren Praxen die Morbidität auch adäquat abzubilden", heißt es in dem Schreiben.

Der Hausärzteverband Hessen begründet seine Ablehnung damit, dass die Richtlinien die Versorgung ver- schlechtern würden und die Patienten unter Umständen zu gläsernen Kunden der Versicherungswirtschaft gemacht werden.

Bei der KV Baden-Württemberg hingegen sieht es anders aus. Dort scheint der anfängliche Widerstand gegen die AKR gebrochen. Auf ihrer Internetseite schreibt die KVBW, dass es ab Mitte Dezember „mittels einer kurz gehaltenen Information Tipps zur rationellen Umsetzung der Neuerungen in der Praxis" geben wird.

KERSTIN MITTERNACHT . 